\title{
Mobile Music Technology: Report on an Emerging Community
}

\author{
Lalya Gaye \\ Future Applications Lab \\ Viktoria Institute \\ 41756 Göteborg, Sweden \\ +46-703800134 \\ lalya@viktoria.se
}

\author{
Lars Erik Holmquist \\ Future Applications Lab \\ Viktoria Institute \\ 41756 Göteborg, Sweden \\ +46-703558500 \\ leh@viktoria.se
}

\author{
Frauke Behrendt \\ Media and Film Studies \\ University of Sussex \\ Brighton, BN1 9 RH, UK \\ +44-7840148789 \\ f.behrendt@sussex.ac.uk
}

\author{
Atau Tanaka \\ Sony CSL Paris \\ 6 , rue Amyot \\ F-75005 Paris, France \\ +33-1-44080512 \\ tanaka@csl.sony.fr
}

\begin{abstract}
The new field of mobile music emerges at the intersection of ubiquitous computing, portable audio technology and NIME. We have held a series of international workshop on this topic with leading projects and speakers, in order to establish a community and stimulate the development of the field. In this report, we define mobile music, and map out the field by reporting on the workshop series and accounting for the stateof-the-art.
\end{abstract}

\section{Keywords}

Mobile music, locative audio, mobile computing, ubiquitous computing, locative media, new interfaces for musical expression.

\section{INTRODUCTION}

Mobile music is a new field concerned with musical interaction in mobile settings, using portable technology. It goes beyond today's portable music players to include mobile music making, sharing and mixing. The core themes of NIME - interaction, interfaces and music, can today be deployed on mobile electronics. While NIME projects have mostly been concerned with stationary concert performance or installations, mobility allows NIME concepts to occupy exterior urban space, and exploit people's movements through it, as well as the heterogeneous space and social dynamics found in those environments. The International Workshops on Mobile Music Technology are the first events that focus on this new field. They have played a key role in the development of mobile music since 2004 and can be regarded as one direction for expansion of the NIME community. This report establishes a definition of mobile music, describes the workshop series, and accounts for the state-of-the-art.

\section{MOBILE MUSIC TECHNOLOGY}

A number of recent technological advances have pushed the envelope of possible human-computer interactions, giving rise to new fields such as locative media and pervasive gaming. Mobile computing enables systems to be used anywhere and on the move. Coupled with context-aware computing and global positioning technology, mobile devices can respond to the

Permission to make digital or hard copies of all or part of this work for personal or classroom use is granted without fee provided that copies are not made or distributed for profit or commercial advantage and that copies bear this notice and the full citation on the first page. To copy otherwise, or republish, to post on servers or to redistribute to lists, requires prior specific permission and/or a fee.

NIME'06, June 4-8, 2006, Paris, France.

Copyright remains with the authors.

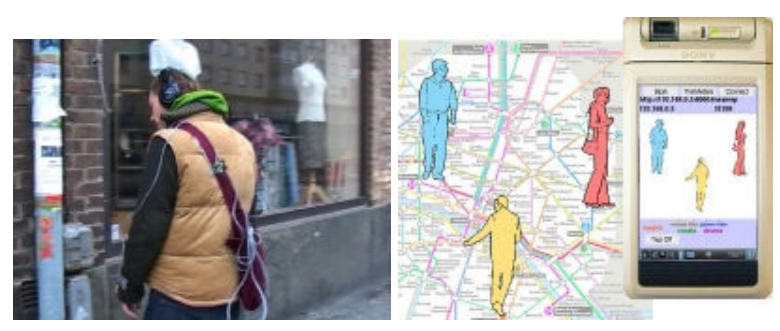

Figure 1: Mobile music projects:

a) Sonic City; b) Malleable Mobile Music.

user's surroundings and location, situating interaction within everyday settings. Augmented and mixed-reality technologies merge the digital and physical realm, making them cohabit in the same environment. Ad hoc, peer-to-peer and distributed networking allow groups of users - ranging from co-located strangers to dislocated friends - to collaborate spontaneously, across distances and without the need for centralised supervision. Meanwhile, the miniaturisation of consumer electronics and improvements in high-capacity digital storage have given rise to powerful portable MP3 players that could easily contain one's complete music collection. Moreover, mobile phones have brought us ubiquitous network connectivity. Mobile music emerges at the crossroads of these technological advances and their resulting new practices, joining the worlds of ubiquitous computing and locative arts, with mobile consumer electronics, and the sensing and interaction tradition of NIME.

\subsection{Mobile Music: Beyond Portability}

Mobile music as a term covers any musical activity using portable devices that are not tethered to a specific stationary locale; in particular those where the activity dynamically follows users and takes advantage of the mobile setting, thereby leveraging novel forms of musical experience. Mobile music devices might possess properties such as context awareness, ad hoc or distributed network connectivity, or location sensing, sometimes combined with technology embedded in the physical environment. Therefore, they can be used anywhere and on the move, and take advantage of people's displacements, location, and of the changes of social and geographical context that mobility implies. Examples of mobile music activities include pushing music to people nearby [16], sonifying local Wi-Fi coverage while riding a bike [19], or remixing music tracks with remote friends across peer-to-peer networks [27]. Mobile music goes beyond the iconic Walkman ${ }^{\mathrm{TM}}$, and does not need to imply individual use, headphones or passive music listening. It spreads over a large spectrum of musical interactions, ranging from consumption to creation, and with mobility increasingly blurring this distinction [11]. Mobile music resonates with practices of both NIME musicians and everyday users of consumer audio products. 


\subsection{Reconsidering Musical Interaction with Mobility}

Mobile music creates a tension between music and place as well as new relationships between musician, listener, and music. For electronic musicians, the mobile environment offers more than just a new place to transplant NIME techniques. Rather, mobility encompasses specificities that encourage us to reconsider the basic tenets of musical interaction. Mobility implies outdoor environments where space and place become tangible parameters, and also implies always-on itinerant devices: location can become a "sensor" input to music systems, people nearby can become part of an ad hoc networked musical performance. With networked multi-user systems, mobility allows musical engagement beyond eye-to-eye contact. It also asks the NIME musician to consider social aspects in everyday public space, an environment not primarily dedicated to music use and where people might already be involved in a number of adjacent and simultaneous activities.

\subsection{Another Dimension to Creative Engagement with Consumer Products}

Many mobile music projects draw on earlier popular electronic music movements such as remix- and DJ-culture, file-sharing or playlists. They extend creative ways of engaging with portable consumer audio technology by weaving them into everchanging geographical and social contexts. One example is tunA [4], where people in close proximity can share music by listening to each other's MP3 playlists, getting a taste of people's musical preferences across various social situations. There is a broad range of possibilities in terms of making music with mobile consumer devices, from ringtones, to mobile soundscape recording or sound art. Widespread platforms such as mobile phones are used as musical instruments and interfaces, encouraging the public to explore new ways of looking at their personal mobile devices. Projects working with such communication technologies invite musicians and lay people alike to participate in performances, group improvisation, sound art or remixing, for example collaborating with strangers in same physical space (e.g. on the bus), or jamming with remote friends while strolling around town.

\section{INTERNATIONAL WORKSHOPS}

At its early stage, mobile music was rapidly gaining popularity and relevance but lacked a clear sense community and an explicit demarcation as a field. As for any emerging field, it was therefore important to establish a community of people who could share experiences and communicate ideas. A good way to achieve such a goal is focused workshops. For instance, the NIME conference series grew out of a workshop at CHI 2001 [23]. In 2004, we started a series of international workshops on the subject in order to establish and develop the field of mobile music. The workshops have gathered a mix of researchers, designers, musicians, new media artists, and representatives of the industry [15]. They have raised awareness about existing projects as well as helped actors of the field with backgrounds in multiple disciplines to identify common goals and issues, share resources, and introduce one another to relevant technologies, methods and concepts. The purposes and formats of the workshops have varied as the community evolves but activities in common include presentations of projects, in-depth discussions, brainstorming sessions and hands-on activities.

The first International Workshop on Mobile Music Technology was organised at the Viktoria Institute in Göteborg, Sweden, in June 2004. The purpose was to gather a number of researchers with a shared interest in mobile music, and to attract additional people who might be interested in making the community grow. This workshop focused on presenting existing projects and defining the field. It had 15 external participants, plus organisers and student volunteers.

The second workshop was organised in May 2005 at NIME 2005 in Vancouver, Canada. This time, the community was better defined, and the workshop time was shared between presentations of new projects, in depth-discussions and handson brainstorming activities. It attracted 18 external participants.

The third edition of the workshop was a two-day event that took place in March 2006 at the University of Sussex, Brighton, UK. It gathered nearly 30 participants and focused on the locative media aspect of mobile music, with presentations by invited speakers, feedback sessions about work-in-progress projects, and hands-on activities with the latest mobile music technology.

\subsection{Projects}

The workshops feature state-of-the-art mobile music projects, in the form of presentations by guest speakers and peer-reviewed papers, posters and demonstration sessions (see Figure 2.a), as well as feedback sessions for works-in-progress. These projects are at the centre of the field's development and demonstrate its diversity and potential. Many use generic mobile platforms such as mobile phones or handheld computers; others use hacked or custom-made technology to better respond to specific needs and requirements. All have in common taking advantage of the mobile nature of mobile technologies and situations as an intrinsic part of their work. Projects can be grouped along the following emerging themes.

Several projects explore collaborative music making with mobile technology. Malleable Mobile Music [28] (see Figure $1 . b$ ) is a location-based and peer-to-peer networked remixing system. TGarden [24] is an interactive environment for theatrical music making using wearables. Sequencer404 [17] allows multi-user control of a musical sequencer through telephony and Voice over Internet Protocol (VoIP). In CELLPHONIA [8], people engage in a location-based mobile phone karaoke opera. The collaborative public art performance China Gates [10] synchronises a set of tuned gongs with GPS as participants follow different routes. Mobile phones are used for interacting with a sound installation in Intelligent Streets [18]. Finally, IMPROVe [29] is a architecture for collaborative improvisation with sounds recorded with mobile devices.

Some of the projects in the genre of mobile music making enable individual users to manipulate sounds and create music by interacting with environmental factors: the physical urban environment with Sonic City [12] (see Figure 1.a), and ambient lighting conditions in Solarcoustics [3]. A mobile user-interface platform for such interactions in a personal area network (PAN) was also demonstrated [30].

Another theme is mobile music listening and sharing. Some projects address the sharing of playlists and music across peerto-peer networks, enabling users to listen to their neighbours' music either synchronously (SoundPryer [21] and tunA [4]) or asynchronously (Push!Music [16]). Other projects transform music albums into narratives spread across geographical space (Location33 [9]), or enable the cultivation of public "sound gardens" located in Wi-Fi connections, as an overlay on physical space (Tactical Sound Garden [TSG] [25]).

A third area is dedicated to HCI and mobile music. It includes SonicPulse [2] - a project providing an acoustic way of 
passively monitoring or actively exploring a shared music space, Music Mood Wheel [1] - an auditive interface for navigating music spaces on the move, and Minimal Attention Navigation via Adapted Music [14] - a musical navigation system for pedestrians.

Meanwhile, some workshop participants have taken a more sociological or media-studies approach, looking at the relation between music taste, use and identity [11], soundscapes and people's everyday experience of place [22], mobile phones and its use in sound-art [5], and mobility, sound and urban culture [7]. These contributions have brought insightful humanistic perspectives for the development of the field of mobile music, grounding it on social realities, aesthetics and already emerging practices.

\subsection{Group Activities}

The workshops included group activities with structured brainstorming sessions in the first two workshops, as well as feedback sessions and hands-on experience of mobile music technology in the third one. These were combined with in-depth discussions on various topics relevant to mobile music and on current issues, opportunities and challenges in the field - e.g. the relationship between mobile devices, space and the body in movement, or how to approach context-aware platforms developed in the field of ubiquitous computing with a NIME perspective.

\subsubsection{Brainstorming Sessions in the $1^{\text {st }}$ Workshop}

An important function of the first workshop was to map out the field and define future directions. We organised a series of structured brainstorming activities that ran over two days. Participants were divided into three groups, each dedicated to one of the following topics: mobile music creation; mobile music sharing; business models and the future of the mobile music industry. In addition to this, we had pre-defined a number of themes to investigate: infrastructure and distribution; genre and formats; social implications; ownership; business models; creativity; interaction and expression; mobility; users and uses.

Each group would choose four themes from the list, and discussed them from the perspective of their overall topic. For instance, the group on Business models discussed the theme Genre and format, raising issues such as length of compositions, use of meta-tags or potential revenue from different kinds of formats. After the first day of brainstorming, results were presented to the other groups. The second day was dedicated to defining design dimensions for mobile music applications based on day one's sessions (for example solo vs. collective, foreground vs. background), and to mapping the emerging design space to existing or future projects. The sessions raised a number of issues, including "in-between" states that are neither mobile nor stationary, how musical taste is used to establish personal identity, to the meaning of ownership and where added value could be elicited.

\subsubsection{Bodystorming Session in $2^{\text {nd }}$ Workshop}

In the $2^{\text {nd }}$ workshop, hands-on activities were kept to one afternoon. They focused on bodystorming of mobile music applications and scenarios. Bodystorming is a method where participants act out a particular scenario of use, taking the roles of e.g. users or artefacts and focusing on the interaction between them [6]. With this method, participants explored various mobile music themes, developed simple application ideas, and physically enacted scenarios of use in order to get an embodied understanding of design challenges and opportunities specific to mobile music.
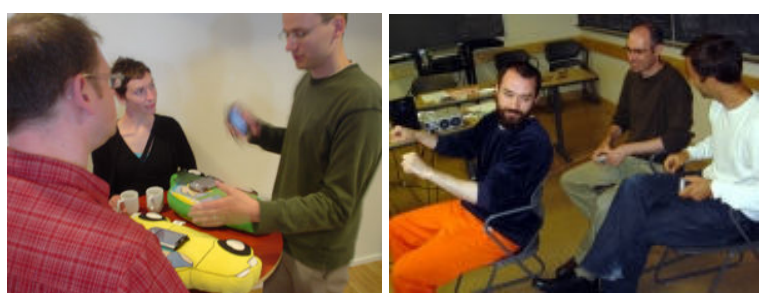

Figure 2: Mobile music workshops: a) Project demonstration and b) scenario bodystorming

Participants first combined randomly chosen instances of the following categories: situations (e.g. driving a car while it snows); users (e.g. school kids); technological infrastructures (e.g. Wi-Fi, GPS); types of music uses (create, share, organise...). Combinations were assigned to each group and developed into 3 application or scenario ideas per group during short brainstorming sessions. Each group decided on one idea and further developed it through bodystorming. Scenarios were then acted out to the rest of the workshop to stimulate discussion. An example of scenario was a bicycle-taxi working as a peer-to-peer server and broadcasting its clients' music on loudspeakers in Kingston, Jamaica (see Figure 2.b). This scenario generated discussions on mobile ways of sharing and outputting music in public space, and of their social adequacy.

\subsubsection{Feedback Sessions and Hands-On Activities in the $3^{\text {rd }}$ Workshop}

On the first day of the third workshop, selected work-inprogress projects grouped in parallel sessions received expert feedback during critical and supporting discussions. Through this participants identified crucial issues and presented their findings to the other groups. The second day was hands-on and gave participants access to technologies for mobile music that they might otherwise not have been exposed to. Participants were given tutorials on sensors for mobile music, and on miniMIXA [20], a mobile music software mixer and mini recording studio for hand-held devices. They were also introduced to socialight [26], an audio space annotation platform for sharing location-based media. As a follow-up, participants sketched out possible applications combining such technologies.

\subsection{Dissemination of Results}

The output from the workshops has been presented in contexts outside of NIME and of the workshop itself. Two of the coauthors moderated a panel discussion at the ACM SIGGRAPH 2005 conference on the subject of Ubiquitous Music [13] where the majority of the panellists selected by the conference had previously participated in the workshops. As the largest international conference on digital media and emerging technologies, the SIGGRAPH panel underscored the pertinence of mobility and musical interaction to a wider field. Authors have also given lectures about mobile music in art and design schools. Currently, the results from the first workshop are being edited into a book that will be a key reference emphasising the creative potential of mobile music technologies.

\section{CONCLUSIONS AND FUTURE WORK}

We have presented the field of mobile music, its current stateof-the-art, as well as a workshop series with a decisive influence on its development. During the workshops, a multitude of emerging key topics concerning the socio-cultural, artistic, technological and economical aspects of mobile music have been identified. The overall experience of these events has been 
very positive. Out of the participants has crystallised a core group, which is very active in the field. The community continues to grow, with new people being attracted to each workshop and the number of relevant projects increasing consistently. The future of mobile music is now being shaped by a collective community effort and promises interesting future developments. In order to further extend and consolidate the mobile music community and support these developments, we will continue to organise new workshops and will soon publish a website as a resource for mobile music projects and related publications.

\section{ACKNOWLEDGMENTS}

The $1^{\text {st }}$ workshop was organised by the Viktoria Institute, the $2^{\text {nd }}$ by Viktoria and Sony CSL Paris (in conjunction with NIME'05), and the $3^{\text {rd }}$ workshop was a collaboration between Viktoria, the Universities of Sussex and Salford, the Pervasive and Locative Arts Network (PLAN) and Futuresonic. We wish to thank our workshop co-organiser Drew Hemment, the NIME'05 chairs Tina Blaine and Sidney Fels, as well as the mobile music core group, including among others Arianna Bassoli, Gideon d'Arcangelo, Maria Håkansson, Rob Rampley, Chris Salter and Mattias Östergren. We also warmly thank all the workshop participants, reviewers and student volunteers, for making this series of events successful.

\section{REFERENCES}

[1] Andric, A., Xech P.-L. Music Mood Wheel: Auditive Interfaces for Mobile Music Devices. ${ }^{r d}$ International Workshop on Mobile Music Technology, Brighton, UK (2006).

[2] Anttila, A. SonicPulse: Exploring a Shared Music Space.

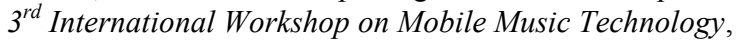
Brighton, UK (2006).

[3] Barnard, M. Solarcoustics: CONNECT. $2^{\text {nd }}$ International Workshop on Mobile Music Technology, NIME'05, Vancouver, Canada (2005).

[4] Bassoli, A., Moore, J., Agamanolis, S. tunA: Synchronised Music-Sharing on Handheld Devices. Adjunct Proc. of Ubicomp'04, Nottingham, UK (2004).

[5] Behrendt, F. Mobile Phone Music. Sound Art and 'Mobile Devices'. $1^{\text {st }}$ International Workshop on Mobile Music Technology, Göteborg, Sweden (2004).

[6] Buchenau, M., Fulton Suri, J. Experience Prototyping. Proc. of DIS'00, ACM Press (2000).

[7] Bull, M. The World According to Sound: Investigating the World of Walkman Users. New Media and Society. Sage London (2001).

[8] Bull, S., Gresham-Lancaster, S., Perkis, T. CELLPHONIA: In The News / Work-In-Progress. $3^{\text {rd }}$ International Workshop on Mobile Music Technology, Brighton, UK (2006).

[9] Carter, W., Liu, L. S. Location 33: A Mobile Musical. $2^{\text {nd }}$ International Workshop on Mobile Music Technology, NIME'05, Vancouver, Canada (2005).

[10] Clay, A., Majoe, D. China Gates. $3^{\text {rd }}$ International Workshop on Mobile Music Technology, Brighton, UK (2006).

[11] D'Arcangelo, G. The New Cosmopolites: Activating the Role of Mobile Music Listeners. $2^{\text {nd }}$ International
Workshop on Mobile Music Technology, NIME'05, Vancouver, Canada (2005).

[12] Gaye, L., Mazé, R., Holmquist, L. E. Sonic City: The Urban Environment as a Musical Interface. Proc. of NIME'03, Montreal, Canada (2003).

[13] Holmquist, L. E., Tanaka, A. Ubiquitous Music: How Are Sharing, Copyright, and Really Cool Technology Changing the Roles of the Artist and the Audience? SIGGRAPH 2005, Los Angeles, USA (2005).

[14] Hunt, R., Apperley, M., Cunningham S. J., Rogers B., Jones, M. Minimal Attention Navigation via Adapted Music. $3^{\text {rd }}$ International Workshop on Mobile Music Technology, Brighton, UK (2006).

[15] International Workshops on Mobile Music Technology http://www.viktoria.se/fal/events/mobilemusic/

[16] Jacobsson, M., Rost, M., Håkansson, M., Holmquist, L.E. Push!Music: Intelligent Music Sharing on Mobile Devices. Adjunct Proc. of UbiComp 2005, Tokyo, Japan (2005).

[17] Jimison, D., Thatcher, T. Sequencer404: A Networked Telephonic Composer. $3^{\text {rd }}$ International Workshop on Mobile Music Technology, Brighton, UK (2006).

[18] Lörstad, H., d'Inverno, M., Eacott, J. The Intelligent Street. $I^{\text {st }}$ International Workshop on Mobile Music Technology, Göteborg, Sweden (2004).

[19] McCallum, D. N. G. Warbike - A Mobile Artistic Sonification of Wireless Networks. Master's thesis in Art and Technology, IT University of Göteborg, Sweden (2005).

[20] miniMIXA. http://www.sseyo.com/products/miniMIXA/

[21] Östergren, M., Juhlin, O. Sound Pryer: Truly Mobile Joint Listening. ${ }^{\text {st }}$ International Workshop on Mobile Music Technology, Göteborg, Sweden (2004).

[22] Phillips, A. Composing the Soundscape: Re-Engaging with Place. $3^{\text {rd }}$ International Workshop on Mobile Music Technology, Brighton, UK (2006).

[23] Poupyrev, I., Lyons, M. J., Fels, S., Blaine, T. New Interfaces for Musical Expression. Extended Abstracts of CHI '01, Seattle, USA (2001).

[24] Ryan, J., Salter, C. TGarden: Wearable Instruments and Augmented Physicality. Proc. of NIME'03, Montreal, Canada (2003).

[25] Shepard, M. Tactical Sound Garden [TSG] Toolkit. $3^{\text {rd }}$ International Workshop on Mobile Music Technology, Brighton, UK (2006).

[26] Socialight. http://socialight.com/

[27] Tanaka, A., Tokui, N., Momeni, A. Facilitating Collective Musical Creativity. Proc. ACM Multimedia, Singapore (2005).

[28] Tanaka, A. Mobile Music Making. Proc. of NIME'04, Hamamatsu, Japan (2004).

[29] Widerberg, R., Hasan, Z. IMPROVe: Mobile Architecture for Sonic Socio-Cultural Exchange. $3^{\text {rd }}$ International Workshop on Mobile Music Technology, Brighton, UK (2006).

[30] Yamauchi, T., Iwatake, T. Mobile User Interface for Music. $2^{\text {nd }}$ International Workshop on Mobile Music Technology, Vancouver, Canada (2005). 